\title{
Effective anisotropic velocity model from surface monitoring of microseismic events
}

\author{
Yang Zhang ${ }^{1 *}$, Leo Eisner², William Barker ${ }^{3}$, Michael C. Mueller $^{3}$ \\ and Kevin L. Smith ${ }^{4}$ \\ ${ }^{1}$ Department of Geological Sciences, University of Michigan, 1100 North University Ave, Ann Arbor, MI 48109, USA ${ }^{2}$ Institute of Rock \\ structure and Mechanics, Academy of Sciences of the Czech Republic, Prague, Czech Republic ${ }^{3}$ Microseismic Inc., 1300 W Sam Houston \\ Pkwy, Suite 200, Houston, TX 77042, USA ${ }^{4}$ Encana Corporation 1800, 855 - 2nd Street SW P.O. Box 2850, Calgary, Canada
}

Received May 2011, revision accepted October 2012

\begin{abstract}
We develop a methodology to obtain a consistent velocity model from calibration shots or microseismicity observed on a buried array. Using a layered 1D isotropic model derived from checkshots as an initial velocity model, we invert P-wave arrival times to obtain effective anisotropic parameters with a vertical axis of symmetry (VTI). The nonlinear inversion uses iteration between linearized inversion for anisotropic parameters and origin times or depths, which is specific to microseismic monitoring. We apply this technique to multiple microseismic events from several treatments within a buried array. The joint inversion of selected events shows a largely reduced RMS error indicating that we can obtain robust estimates of anisotropic parameters, however we do not show improved source locations. For joint inversion of multiple microseismic events we obtained Thomsen anisotropic parameters $\epsilon$ of 0.15 and $\delta$ of 0.05 , which are consistent with values observed in active seismic surveys. These values allow us to locate microseismic events from multiple hydraulic fracture treatments separated across thousands of metres with a single velocity model. As a result, we invert the effective anisotropy for the buried array region and are able to provide a more consistent microseismicity mapping for past and future hydraulic fracture stimulations.
\end{abstract}

Key words: Anisotropy, Microseismicity, Inversion.

\section{INTRODUCTION}

Hydraulic fracture treatments are routinely optimized with microseismic monitoring (e.g., Duncan and Eisner 2010; Maxwell et al. 2010). The characterization of induced microseismic events is crucially dependent on utilizing an accurate velocity model (e.g., Bardainne and Gaucher 2010; Grechka and Duchkov 2011). Traditionally microseismic monitoring has been carried out with temporarily deployed monitoring

*E-mail: yzhan@umich.edu arrays, either deployed in a subsurface monitoring well or on the surface. A new type of permanent array deployed with geophones in shallow boreholes (buried array) allows for consistent microseismic mapping among different fracture treatments at a reservoir scale. However, estimated microseismic locations are very sensitive to the velocity model, thus consistent microseismic mapping from buried arrays requires also a consistent velocity model. We have developed a methodology to invert a consistent anisotropic velocity model with a vertical axis of symmetry (VTI) from microseismicity or calibration shots observed on a surface or near-surface (buried) monitoring array. Because the true locations of the microseismic events are unknown, we do not study the improvement of the 
event locations. We show that with a single velocity model we obtain reasonable values of anisotropic parameters, vertical velocity and depths of located microseismic events.

Seismic anisotropy is the dependence of seismic velocity upon the wave propagation direction (Thomsen 1986). Seismic anisotropy has been used widely for improving reservoir imaging (e.g., Tsvankin and Grechka 2006), lithology discrimination (Vernik 2008), characterizing fractures and stresses (e.g., Prioul and Jocker 2009) and monitoring the timelapse changes of producing fields (e.g., Meersman, Kendall and van der Baan 2009). In active seismic, incorporating elastic anisotropy into migration algorithms allows proper positioning of reflectors and further enhances our understanding of regional velocity structures. In passive seismic, subsurface anisotropy is crucial for obtaining accurate locations and stacking of far-offset receivers as shown by Grechka and Duchkov (2011) and Gei et al. (2011). Grechka and Duchkov (2011) derive an inversion algorithm using microseismicity where origin times, velocity model and event locations are inverted simultaneously in borehole monitoring geometry and only the well constrained parameters are updated. Gei et al. (2011) showed a trade-off between inverted parameters in a surface configuration on a synthetic data set in a homogeneous model. In this study we extend the algorithm of Gei et al. (2011) to a 1D medium and apply it to real data set from a buried array.

The current migration-type location from surface monitoring arrays relies on P-wave stacking from vertical geophones (e.g., Duncan and Eisner 2010). A majority of the velocity models are derived from 1D sonic logs, or alternately from VSP or checkshot 1D models. Checkshots or sonic logs provide information on the vertical velocity only with single offset, thus models derived from such data are usually smoothed to a $1 \mathrm{D}$ isotropic layered model. However, such models frequently locate known calibration shots to an inaccurate depth. The discrepancy can be explained by the presence of VTI anisotropy of the 1D layered models. The VTI anisotropy is likely a good model for a shale reservoir. For example, Sayers (1993, 1994) showed that shales can develop strong anelliptic anisotropy due to intrinsic textural properties. In other words, shear and compressional velocities within shales are faster in the horizontal direction than the vertical direction.

Alternatively one may locate the calibration shot to a correct depth by scaling up (or down) the isotropic velocity. This approach may provide a good accuracy of located depths for calibration shots. However, if a perforation shot or microseismic event occurs at a significantly different lateral position, this approximation may produce biased results as receiver statics are compensating for an incorrect velocity model. Furthermore, the scaled velocity model does not reflect real seismic velocities measured in the vertical direction by independent techniques (e.g., checkshots). We will show that VTI anisotropy seems to better reflect seismic velocity than a scaled-up $1 \mathrm{D}$ isotropic velocity profile, resulting in smaller residuals and more consistent receiver statics for multiple treatments.

While active seismic routinely uses anisotropic velocity models for the imaging of subsurface structures, passive seismic surface monitoring is challenged with the additional issue of unknown origin time and location (for microseismic events). To overcome this challenge, surface monitoring uses either velocity models derived from active seismic (e.g., Chambers, Kendall and Barkved 2010) or a model built from calibration shots (Gei et al. 2011). Downhole monitoring of microseismic events uses an additional type of wave, S-waves, which are radiated from microseismic events (e.g., Wuestefeld et al. 2010; Grechka and Duchkov 2011). However, velocity model calibration in downhole monitoring poses a greater challenge due to increased uncertainty in the positioning of sources and receivers (Bulant et al. 2007). Surface monitoring does not have such issue with receiver positioning (with high GPS accuracy and precision). The S-waves are not usually available for velocity model calibration because they are more attenuated than P-waves. Thus we focus on velocity model calibration with P-waves only from surface monitoring. This study extends the homogeneous model calibration of Gei et al. (2011) to a 1D layered model through newly developed methodology assuming weak VTI anisotropy. We test this calibration using a real data set acquired with a buried array and are able to locate microseismic events from multiple hydraulic fractures kilometres apart with one single velocity model.

\section{METHODOLOGY}

\section{Inversion of microseismic data for vertically transversely isotropic media}

We derive a linearized inversion for layered weak VTI anisotropic media with effective anisotropic parameters. By effective anisotropic parameters we mean constant Thomsen parameters from the depth of the induced microseismic events (or calibration shots) to the surface. Although anisotropic parameters are unlikely constant for the whole depth interval, they represent an average value as we are not able to determine the depth stratification of anisotropy (e.g., Bakulin et al. 2010). The vertical stratification cannot be determined as all sources and receivers are at two depth intervals, unlike those in the checkshot or sonic logging measurement. If we 
know in advance that only certain layers are anisotropic, the methodology can be easily generalized for an inversion only in those layers.

In VTI anisotropy the compressional qP-wave velocity depends only on the incidence angle $\Theta$ of the ray, i.e., qP-wave velocity $v_{P}(\Theta)$ is a function of the incidence angle, not the azimuth. In this notation the vertical velocity is $\alpha=v_{P}(\Theta=0)$. The weak VTI anisotropic qP-wave velocity can be approximated as (Thomsen 1986):

$\left.v_{P}(\Theta) \approx \alpha\left[1+\delta \sin ^{2} \Theta+(\epsilon-\delta) \sin ^{4} \Theta\right)\right]$,

Where $\epsilon, \delta$ are Thomsen anisotropic parameters that control the qP-wave velocity. In weak anisotropic media both $\epsilon<<1$ and $\delta<<1$. The anisotropic traveltime corresponding to the phase velocity in homogeneous media can then be computed with further linearization of the above equation. (1) assuming small values of Thomsen anisotropic parameters. The approximation is based on Thomsen (1986) who showed that up to first order for weak anisotropy, the ray velocity in the ray direction is equal to the associated phase velocity in the phase (or slowness) direction (perpendicular to the wavefront). Hence, the straight-ray traveltime approximation is

$$
\begin{aligned}
T_{P}^{p r o p}(\Theta) & =\frac{x}{\alpha}\left[\frac{1}{1+\delta \sin ^{2} \Theta+(\epsilon-\delta) \sin ^{4} \Theta}\right] \\
& \simeq \frac{x}{\alpha}\left[1-\delta \sin ^{2} \Theta-(\epsilon-\delta) \sin ^{4} \Theta\right] \\
& =t^{i s o}\left[1-\delta \sin ^{2} \Theta-(\epsilon-\delta) \sin ^{4} \Theta\right],
\end{aligned}
$$

where $t^{i s o}$ is the traveltime within the layer of isotropic media with velocity $\alpha$ (i.e., vertical velocity) and $x$ is the distance the ray propagates through.

Assuming effective (constant) anisotropic parameters $\delta$ and $\epsilon$ for all layers we can then calculate the traveltime through layered VTI anisotropic media:

$$
\begin{aligned}
T_{P}^{A n i} & =\sum_{i=1}^{z} T_{P_{i}} \\
& =\sum_{i=1}^{z} t_{i}^{i s o}-\delta \sum_{i=1}^{z} \sin ^{2} \Theta_{i} t_{i}^{i s o}-(\epsilon-\delta) \sum_{i=1}^{z} \sin ^{4} \Theta_{i} t_{i}^{i s o} \\
& =T_{P}^{I s o}+\delta A+(\epsilon-\delta) B
\end{aligned}
$$

where $t_{i}^{i s o}$ is the traveltime in isotropic media in the $i^{\text {th }}$ layer, $z$ is the number of layers, $A=-\sum_{i=1}^{z} \sin ^{2} \Theta_{i} t_{i}^{i s o}$ and $B=-\sum_{i=1}^{z} \sin ^{4} \Theta_{i} t_{i}^{i s o}$. Note that in weak VTI media the ray trajectory does not depend on $\epsilon$ or $\delta$ (to the first order) and we can use the ray trajectory computed in an isotropic (layered) model. Both A and B are functions of source location as well as receiver position for each event.

To invert for anisotropic parameters we need to compare arrival times (picks of qP-wave arrivals) with anisotropic traveltimes adjusted with the origin times. Microseismic events depths are usually not known with sufficient accuracy, which will affect the inversion results. The difference between arrival time $T_{i j}^{\text {Pick }}$ in the $j^{\text {th }}$ receiver from the $i^{\text {th }}$ event is used to calculate residual $R_{i j}$ :

$$
\begin{aligned}
R_{i j}= & T_{i j}^{P i c k}-T_{i j}^{A n i}-T_{0_{i}}=T_{i j}^{\text {Pick }}-T_{i j}^{I s o} \\
& -\delta A_{i j}(\epsilon-\delta) B_{i j}-T_{0_{i}},
\end{aligned}
$$

Where $T_{i j}^{A n i}$ is the anisotropic traveltime from the $j^{\text {th }}$ receiver of the $i^{t h}$ event, $T_{0_{i}}$ is the origin time of the $i^{\text {th }}$ event and $A_{i j}$ and $B_{i j}$ are the coefficients corresponding to $\mathrm{A}$ and $\mathrm{B}$ defined above for the $i^{t h}$ event and the $j^{t h}$ receiver. Note that the inversion requires knowledge of the origin times as this parameter has a trade-off with depth and relative moveout and will impact the calculation of the anisotropic parameters. Hence origin times need to be included in the inversion.

To minimize overall residuals in the least square norm, we need to minimize $\sum_{i=1}^{n} \sum_{j=1}^{N_{r e c}} R_{i j}^{2}$, which can be rewritten as

$$
\begin{aligned}
& \left(\sum_{i=1}^{n} \sum_{j=1}^{N_{r e c}} R_{i j}^{2}\right) /\left(N_{r e c} n\right)=\left(\sum _ { i = 1 } ^ { n } \sum _ { j = 1 } ^ { N _ { r e c } } \left(-A_{i j} \delta-B_{i j}(\epsilon-\delta)\right.\right. \\
& \left.\left.+D_{i j}\right)^{2}\right) /\left(N_{r e c} n\right)
\end{aligned}
$$

where $D_{i j}=T_{i j}^{\text {Pick }}-T_{i j}^{I s o}-T_{0_{i}}$, and $n$ and $N_{r e c}$ are the number of events and receivers, respectively. The minimum of residuals in equation (5) can be found by solving the set of linear equations for events $i=1, \ldots, n$ :

$\mathrm{d}=\mathrm{Gm}$,

where

$$
\mathbf{m}=\left[\begin{array}{c}
\delta \\
\epsilon-\delta
\end{array}\right], \mathbf{G}=\left[\begin{array}{c}
-A_{11},-B_{11} \\
\vdots \\
-A_{1 j},-B_{1 j} \\
-A_{21},-B_{21} \\
\vdots \\
-A_{n 1},-B_{n 1} \\
\vdots \\
-A_{n j},-B_{n j}
\end{array}\right] \mathbf{d}=\left[\begin{array}{c}
-D_{11} \\
\vdots \\
-D_{1 j} \\
-D_{21} \\
\vdots \\
-D_{n 1} \\
\vdots \\
-D_{n j}
\end{array}\right] \text {. }
$$

This relation can be solved by a least squares inversion of $\mathrm{d}$. If we have the origin time and event location the least squares solution of equation (6) provides an estimate of the 
anisotropic parameters. Thus, we initially estimate the location and origin time and they will iteratively converge to a solution. We show later that this is a correct solution for well constrained vertical velocity and the starting depth is close to the correct depth. To begin the iteration, we need an initial estimate of event locations and event origin times to calculate initial values of $D_{i j}$. The initial origin time can be estimated from a least squares fit to the arrival times

$T_{0_{i}}=\sum_{j=1}^{N_{r e c}}\left(T_{i j}^{P i c k}-T_{i j}^{A n i}\right) / N_{r e c}$.

This derivation of a generalized inversion proposed by Bulant et al. (2007) was originally formulated for a homogeneous medium.

\section{Implementation for microseismic data}

To invert anisotropic parameters we pick qP-wave arrival times on receivers on which we can observe distinct arrivals of direct qP-waves. Our initial velocity model is a layered 1D isotropic model that can be derived from an active surface seismic survey or checkshot velocity. We keep the lateral position of the event located in the isotropic velocity model fixed. However, in principle the horizontal position could also be updated. The depth of the microseismic event can be initially fixed to the depth of fracturing or some other estimates (e.g., depth of location from the isotropic velocity model). In the case of the perforation or calibration shot the position is fixed to a known location. Thus we can use equation (7) to obtain an initial estimate of the origin time of each event with the initial isotropic model. We then iteratively compute the effective anisotropic parameters and origin times (or both origin times and depths) while minimizing the arrival time residuals.

The flow chart in Fig. 2 illustrates a case with known depth of a microseismic event (e.g., a calibration shot) where we apply an iterative inversion for origin times and anisotropic parameters. The initial model has $\epsilon$ and $\delta$ set to 0 . By adjusting the origin time of every event with equation (7), we can iteratively update the anisotropic velocity model and origin times. We continue adjusting the anisotropic parameters by equation (6) and origin times by equation (7) until the overall residuals calculated by equation (5) stop decreasing or are below a certain threshold (e.g., several samples).

In the case of the microseismic events (e.g., events induced by hydraulic fracturing), since we do not know the depth of the events, we should add an additional grid search for depths of the microseismic events. However, we found that such an inversion is less stable (see also Gei et al. 2011) and therefore we update the depth of only one event at a time instead of searching for depths of all the events simultaneously, as illustrated in the flow chart of Fig. 3. While the previous inversion with known depth and vertical velocity had only trivial computation cost, the algorithms including depth search and vertical velocity inversion are more expensive to compute, even though it is still feasible to compute on a PC computer.

The inversion procedures illustrated above are applicable to different cases. Inversion in Fig. 2 is suitable for the inversion of calibration shots with known depths (e.g., string shots or perforation shots). However, in some formations calibration shots have a smaller signal than microseismic events. In such cases we use microseismic events to invert for depth in addition to anisotropy and origin times as illustrated in Fig. 3.

\section{TESTS ON SYNTHETIC DATA SETS}

In this section we will investigate the numerical stability of the inversion on synthetic data sets. We performed tests on synthetic data sets simulating the case of constrained depth (e.g., calibration shots) as well as unknown depth (e.g., microseismic events). We finally also test inversion of average vertical velocity with constrained depths (e.g., sonic log with calibration shots). All model inversions started with the same 1D isotropic vertical velocity model shown in Fig. 1(c). This model was derived from checkshot measurements. The three tests are:

1) Inversion for $\epsilon$ and $\delta$ iteratively and origin times estimated, assuming a correct 1D isotropic model and event depths.

2) Inversion for $\epsilon$ and $\delta$ iteratively, origin times and event depths are estimated, assuming a correct $1 \mathrm{D}$ isotropic vertical velocity.

3) Inversion for $\epsilon$ and $\delta$ iteratively, origin times and scaling factor for $1 \mathrm{D}$ isotropic vertical velocity are estimated, assuming correct event depths.

We use the receiver geometry of the case study discussed in Fig. 1. Figure 1(a) shows a map view of the array, while Fig. 1(b) shows the cross-section view of the array, both with raypaths plotted. All noise-free arrival times are computed with the effective (constant) anisotropic parameters in the 1D layered model of vertical velocities shown in Fig. 1(c). Specifically, the velocity model used is the same for all tests with constant values of $\epsilon=0.1$ and $\delta=0.05$. The receiver geometry shown in Fig. 1(a) consists in 101 receivers buried at an approximate depth of $72 \mathrm{~m}$ below the free surface. We performed the inversion algorithm on different sets of microseismic events with distributions derived again from the 
(a)
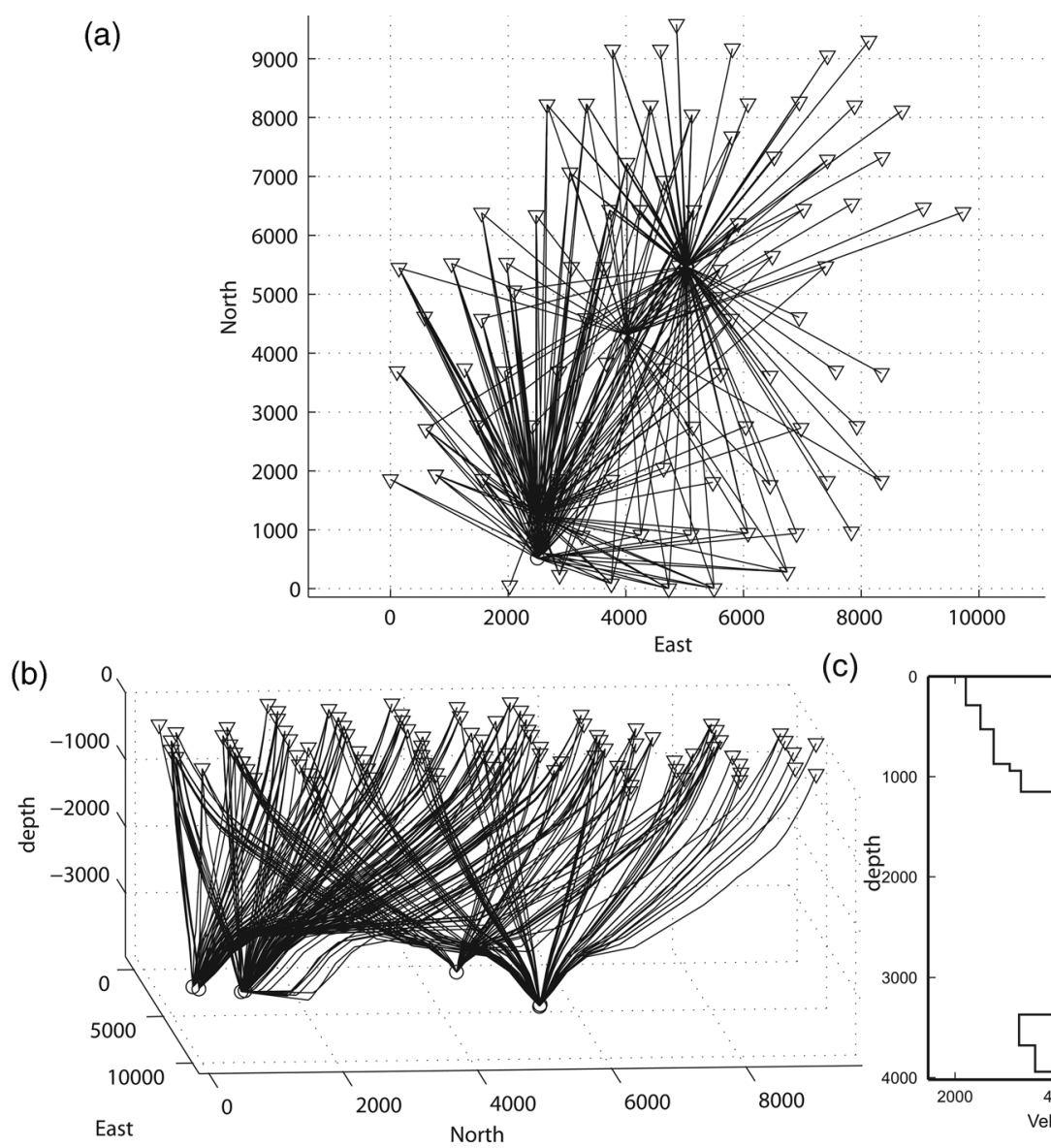

(c)

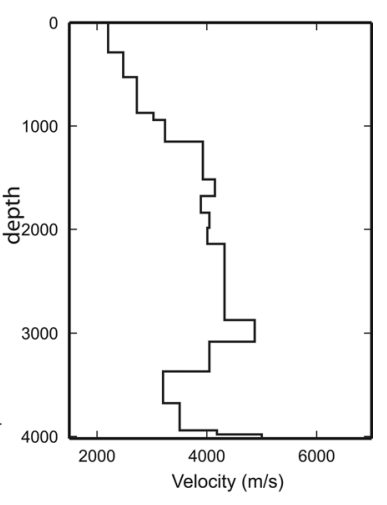

Figure 1 (a) Mapped projected locations of 101 stations (triangles) of the array with raypaths for all picked arrival times. A total of 343 raypaths are shown. (b) 3D view of the raypaths from the east. (c) The initial vertical velocity profile with 17 vertical layers.

Table 1 Locations of the events (local coordinates are also shown in Fig. 1)

\begin{tabular}{lllll}
\hline Events & $\begin{array}{l}\text { Easting } \\
\text { [metres] }\end{array}$ & $\begin{array}{l}\text { Northing } \\
\text { [metres] }\end{array}$ & $\begin{array}{l}\text { Depth } \\
\text { [metres] }\end{array}$ & $\begin{array}{l}\text { Number of picks } \\
\text { in the case study }\end{array}$ \\
\hline E1_1 & 4019 & 4310 & 3482 & 53 \\
E2_1 & 5036 & 5456 & 3838 & 59 \\
E2_2 & 5015 & 5456 & 3862 & 33 \\
E3_1 & 2471 & 1240 & 3950 & 54 \\
E3_2 & 2496 & 1293 & 3862 & 31 \\
E3_3 & 2502 & 515 & 3864 & 47 \\
E3_4 & 2605 & 597 & 3879 & 34 \\
E4_1 & 4043 & 4208 & 3930 & 32 \\
\hline
\end{tabular}

case study listed in Table 1 . In the case study we used events from four different stimulation programs. The naming convention for these events is EX_Y, where $\mathrm{X}$ is the number of the stimulation program and $\mathrm{Y}$ is the number of events in that program. Stimulation 3 is in the southern part of the array and the remaining stimulations 1, 2 and 4 are in the central part of the array. From these events we selected several sets of events for testing:

(Set 1) Single event inversion from the centre of the array (E1_1).

(Set 2) Single event inversion from the south of the array (E3_1).

(Set 3) Events inversion from three central positions (E1_1, E2_1, E2_2).

(Set 4) Events inversion from three southern positions in the array (E3_1, E3_2, E3_3).

(Set 5) Events inversion from all events.

(Set 6) Three events representing the central parts (E2_1, E2_2) and the southern part of the array (E3_1).

Since the true model is known, the synthetic tests allow us to quantify the algorithm's ability to recover the Thomsen anisotropic parameters in the particular geometry of our case 
Table 2 Sensitivity test on synthetic data sets with a variable number of events of known depths (with and without added noise). Noise level is the standard deviation of random time perturbation added to computed traveltimes. Input (true) values are $\epsilon=0.1, \delta=0.05$ and origin times were set to -0.2 seconds

\begin{tabular}{|c|c|c|c|c|}
\hline$\epsilon$ & $\delta$ & $\begin{array}{l}\text { Origin Time } \\
\text { (s) }\end{array}$ & $\begin{array}{l}\text { Events } \\
\text { used }\end{array}$ & $\begin{array}{l}\text { Noise Level } \\
\text { (ms) }\end{array}$ \\
\hline 0.1 & 0.05 & -0.2 & Set 1 & 0 \\
\hline 0.1 & 0.05 & -0.2 & Set 2 & 0 \\
\hline 0.1 & 0.05 & $-0.2,-0.2,-0.2$ & Set 3 & 0 \\
\hline 0.1 & 0.05 & $-0.2,-0.2,-0.2$ & Set 4 & 0 \\
\hline 0.1 & 0.05 & -0.2 all & Set 5 & 0 \\
\hline 0.1 & 0.05 & $-0.2,-0.2,-0.2$ & Set 6 & 0 \\
\hline 0.1 & 0.05 & $-0.2,-0.2,-0.2$ & Set 6 & 4 \\
\hline 0.1 & 0.04 & $-0.2,-0.2,-0.2$ & Set 6 & 8 \\
\hline 0.1 & 0.06 & $-0.2,-0.2,-0.2$ & Set 6 & 16 \\
\hline 0.13 & 0.04 & $-0.22,-0.21,-0.22$ & Set 6 & 32 \\
\hline
\end{tabular}

study and test the sensitivity to different input parameters. We can compute the obtained anisotropic parameters $\epsilon$ and $\delta$ and determine the accuracy of the inverted origin times.

\section{Sensitivity to event distribution and noise in arrival times}

Gei et al. (2011) carried out extensive numerical testing of surface array design and noise sensitivity on the inversion. They showed that the uncertainty of the inverted parameters is sensitive to a particular source-receiver geometry. In this section we study the proposed $1 \mathrm{D}$ generalization on specific geometries of the case study of this article. We assume that the microseismic event signal is detected on all receivers. In the first test we assume we have various calibration shots with known depths at different parts of the reservoir.

Table 2 shows the inversion results for noise-free data with five sets of events and results with noise contaminated arrivals using Set 6. Obviously the noise-free arrival times provide highly accurate results up to two decimal places, although differences show up on the fourth decimal places. A larger number of events constrained the model better by reducing the differences. Interestingly the single event in the southern part of the reservoir constrains the VTI model equally well as the event in the centre of model.

On the set of eight events, Gaussian random noise was added to traveltimes simulating picking errors. The picking

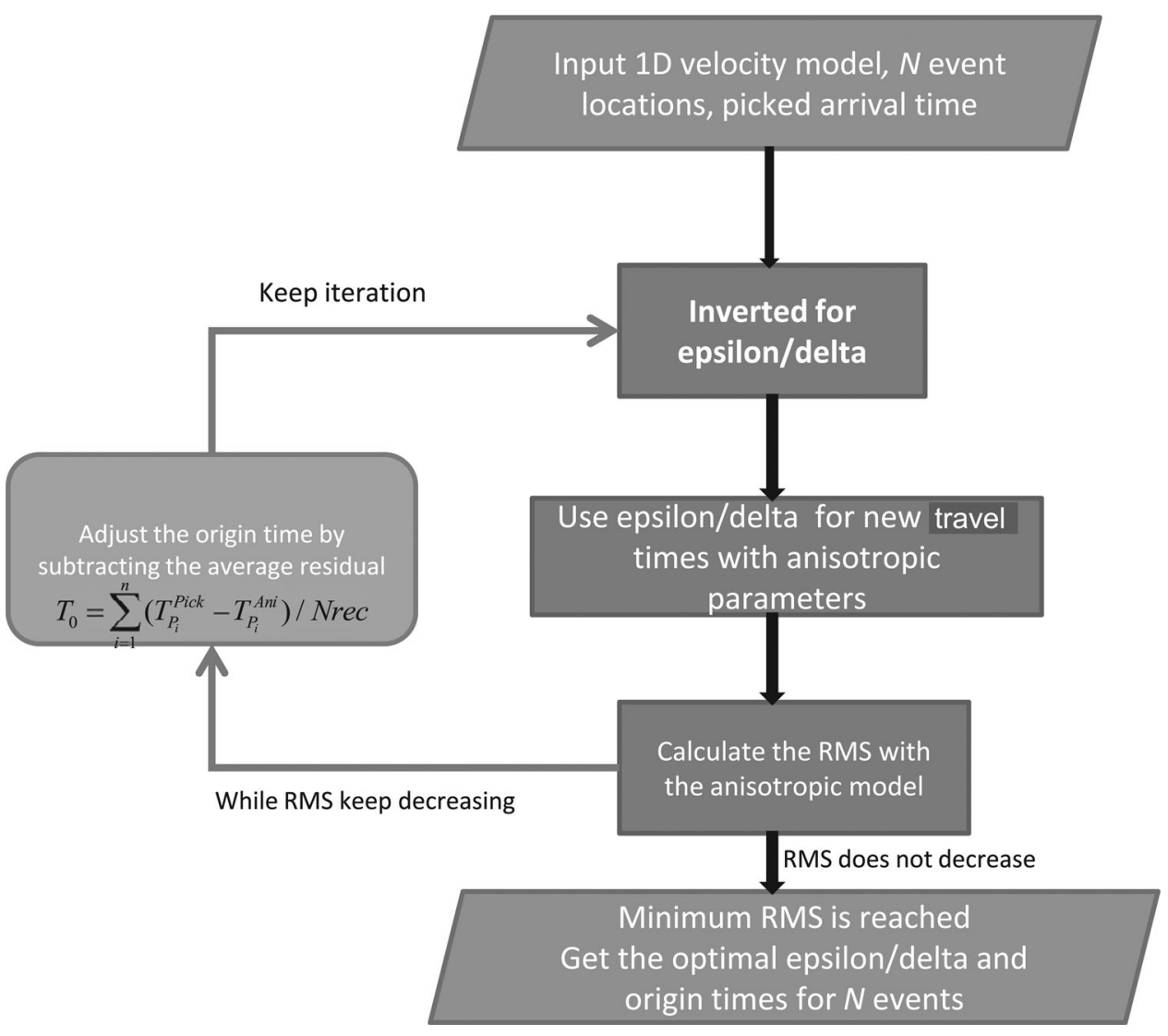

Figure 2 Flow chart of the algorithm used for the inversion of origin time and anisotropic parameters. 
errors may result from high-noise levels or simply local velocity perturbations. In the case study, the arrival picks of events originally had an average root mean square (RMS) of time residuals larger than $30 \mathrm{~ms}$. Thus, we perturbed the arrival times by Gaussian noise with a standard deviation varying from 4-32 ms. Generally, we can observe a decreasing precision of the inverted parameters as the noise levels increase. However, the errors of the inverted parameters do not seem to be significantly high. Even with $16 \mathrm{~ms}$ of noise level in arrival times, $\epsilon$ and origin times are accurately inverted and $\delta$ is less than $20 \%$ different from the input value. This observation is in good agreement with Gei et al. (2011) who observed also only 1-2\% errors in inverted anisotropic parameters with noise levels up to $4 \mathrm{~ms}$. In conclusion, inversion of noise contaminated arrival times does not seem to be significantly affected up to the noise level of a standard deviation of $8 \mathrm{~ms}$.

\section{Sensitivity to source depths}

Microseismic events have unknown depths. Even depths of calibration shots may not be known well due to deviation survey uncertainty. Hence, to study the sensitivity of the inverted parameters to depth, we vary the source depths of the synthetic microseismic events by $5-20 \%$ and evaluate the effect of the unknown (or biased for perforation shot) depths. The purpose of the synthetic test is to understand the sensitivity of the obtained anisotropic parameters to depths of the microseismic events. The incorrect depths of microseismic events result in a strong bias of the inverted anisotropic parameters $\epsilon$ and $\delta$ (see also Gei et al. 2011). If the assumed depths of microseismic events are greater than the true depths, $\epsilon$ and $\delta$ increase while the origin time shifts to later time (absolute) relative to the true origin time. The shifts are apparently large but the increased anisotropic parameters flatten more moveout across the array, thus shifting the origin time to later times reduces the misfit of travel and arrival times. For a $5 \%$ change in the source depth, $\epsilon$ changes by $15 \%$ and $\delta$ changes by $40 \% . \delta$ is more sensitive to the incorrect depth than $\epsilon$, as $\delta$ plays a more important role in near-to-middle offsets (and traveltimes are matched to measured arrival times). Luckily, $5 \%$ is perhaps the upper estimate for an error in the depth position of a calibration shot due to a deviation survey in a long lateral well (see Bulant et al. 2007 for more details). However, in the case of microseismic events used for velocity model calibration, a depth error of $5 \%$ is quite possible.

In some cases vertical velocity profiles may not be known with sufficient accuracy. For example, typically sonic logging over a limited depth interval is available and the velocity in the depth interval above the sonic log is extrapolated to the surface. This may result in vertical velocity profile errors, which may correspondingly result in incorrectly estimated anisotropic parameters. As the inversion technique is sensitive only to an average vertical velocity we tested the sensitivity by using different scaling factors on the vertical velocity profile (see Fig. 1c) to obtain the vertical input velocity profile for the inverse problem.

We vary the input vertical velocity profile by increasing the average vertical velocity to $105-120 \%$ of the original profile. The results show that changing the vertical velocity strongly affects both the anisotropic parameters and the origin time. Contrary to depth change, $\epsilon$ is more sensitive to velocity change. For example, a $5 \%$ increase in vertical velocity causes a $30 \%$ increase in $\epsilon$ and a $25 \%$ increase in $\delta$. The results show large discrepancies of the anisotropic parameters. We note that the RMS errors with incorrect depth or velocity are relatively small as observed also by Gei et al. (2011) but the origin time is significantly shifted. This results from the fact that in surface locations based on P-wave arrivals the origin times compensated partly for errors in the model or source depth. We conclude that the vertical velocity needs to be determined very accurately. If none of the parameters are fixed accurately, it is not possible to determine independently the vertical velocity, source depths and anisotropic parameters in simultaneous inversion.

\section{Additional inversion for source depths or vertical velocity}

In this section we try to assess the possibility of inverting simultaneously for the Thomsen anisotropic parameters, origin times and source depths as discussed in the previous section (see Fig. 3). As shown in the flow chart, the source depths of events are updated successively and iterations are processing with the anisotropic parameters and origin times inverted until minimal RMS is reached. The test is carried out for two events, E1_1 and E2_1, from the central part of the array with different depths. Table 3 shows the initial depths of the two synthetic events set to an approximate depth of injection at $3600 \mathrm{~m}$. The source depths are grid searched with grid step of $30 \mathrm{~m}$ and for noise-free arrival times the final depths for these two events are $3840 \mathrm{~m}$ and $3470 \mathrm{~m}$, which are close to the actual depths. The parameters $\epsilon$ and $\delta$ and origin times are well inverted. However, we found that with a significantly incorrect starting depth the algorithm was not able to find correct depths and did not converge. Thus the choice of event depths, which are close to their true depths, is crucial for successful 


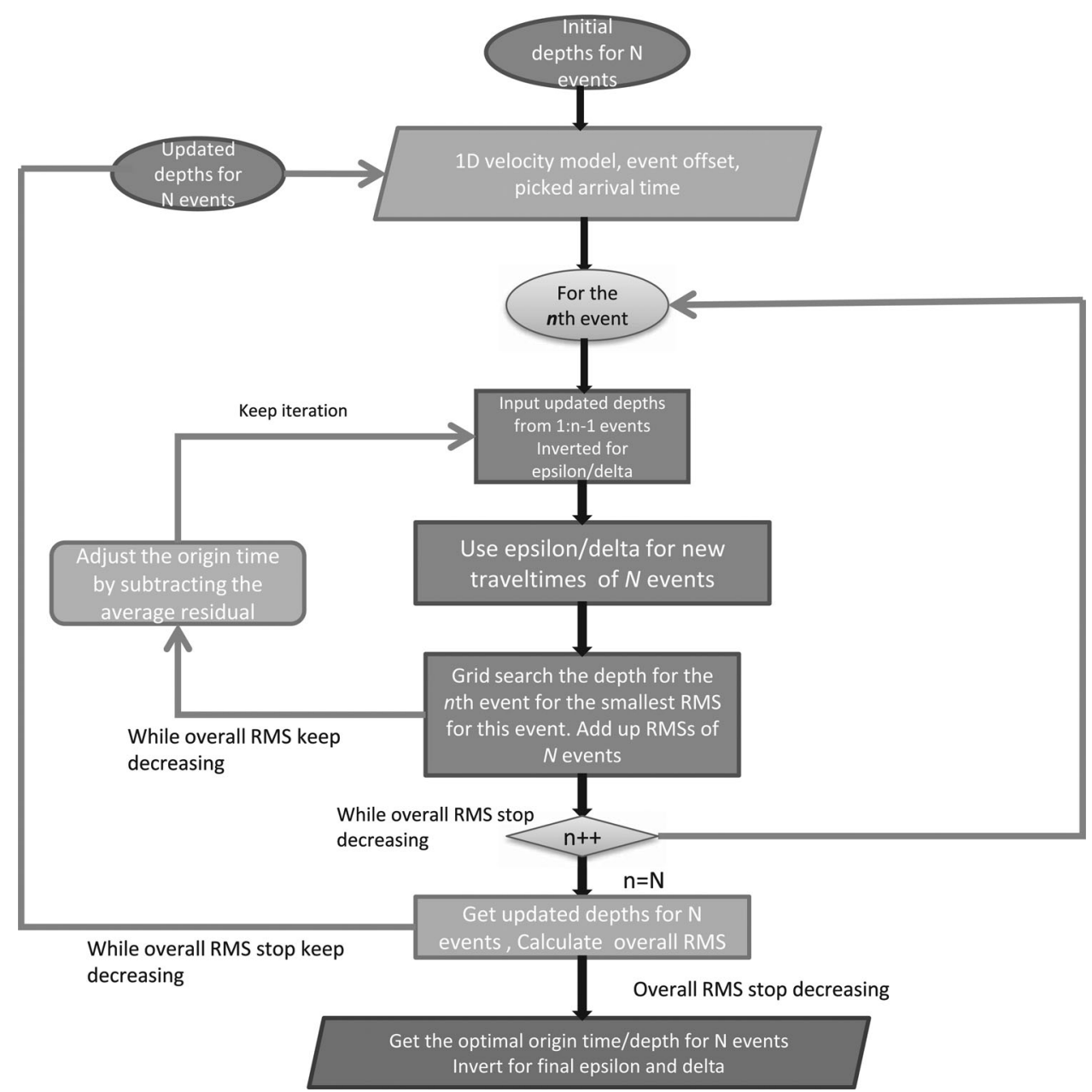

Figure 3 Flow chart of the algorithm used for the inversion of origin time, source depths and anisotropic parameters.

inversion. Furthermore, we test the algorithm with different levels of noise added to the synthetic arrival times starting with no noise, a noise level of $4 \mathrm{~ms}$ (standard deviation of the added noise) and a noise level of $8 \mathrm{~ms}$. Inverting the final depths, Thomsen anisotropic parameters and origin times shows that the picking error does not seem to significantly destabilize the inversion as these inverted depths are within one grid point of their true values.

\section{CASE STUDY}

We apply the inversion of the Thomsen anisotropic parameters (with depths and origin times) to several hydraulic fracturing treatments within a buried array. The array consists in 101 stations and occupies approximately 67 square kilometres (25 square miles), with a station offset spacing approximately $900 \mathrm{~m}$ as illustrated in Fig. 1(a,b). The geophones in each

Table 3 Feasibility of inversion for source depths. Two calibration events were considered at depths 3838 m (E2_1) and 3482 m (E1_1) in effective VTI anisotropy of $\epsilon=0.1$ and $\delta=0.05$. Origin time for both events was set to -0.2 seconds

\begin{tabular}{|c|c|c|c|c|c|c|}
\hline $\begin{array}{l}\text { Initial depth } \\
(\mathrm{m})\end{array}$ & $\begin{array}{l}\text { Final depth } \\
(\mathrm{m})\end{array}$ & $\epsilon$ & $\delta$ & $\begin{array}{l}\text { T0 } \\
\text { (s) }\end{array}$ & $\begin{array}{l}\text { RMS } \\
\text { (ms) }\end{array}$ & $\begin{array}{l}\text { noise level } \\
(\mathrm{ms})\end{array}$ \\
\hline 3600,3600 & 3780,3470 & 0.1 & 0.05 & $-0.20,-0.20$ & 0,0 & 0 \\
\hline 3600,3600 & 3780,3500 & 0.11 & 0.04 & $-0.19,-0.19$ & 7,7 & 4 \\
\hline 3600,3600 & 3810,3500 & 0.12 & 0.02 & $-0.20,-0.19$ & 16,16 & 8 \\
\hline
\end{tabular}


station are buried 60-90 m below the surface and cemented to increase the coupling with the formation. The approximate depth of the stimulated shale is $3800 \mathrm{~m}$, resulting in a maximum offset-to-depth ratio of 2 for events in the south of the array. The depth resulted in a very low signal-to-noise ratio for perforation or calibration shots at known positions. Thus, we applied the inversion to eight high signal-to-noise microseismic events. The eight events are from four different stimulations located in the central and southern part of the array as shown in Fig. 1(a). The coordinates and number of reliably picked arrivals for each event are shown in Table 1. We manually picked arrival times of $\mathrm{P}$-waves ( $\mathrm{qP}$-waves but we shall use $\mathrm{P}$-waves nomenclature in the following) on approximately 50-60 receivers for each event. Some receivers close to the $\mathrm{P}$-wave nodal plane possessed a signal-to-noise ratio that is too low to be picked with confidence.

Table 4 shows the $\epsilon$ and $\delta$, origin time corrections and RMS for each event. The isotropic velocity model was derived from checkshot measurements determined in a nearby well. This $1 \mathrm{D}$ isotropic velocity model was not perturbed when inverting for anisotropic parameters as it represents an ideal vertical velocity measurement in the frequency range relevant to microseismic data assuming high-quality checkshot arrival picks. The origin time correction is a time-shift between the origin time inverted in the starting isotropic velocity model and the origin time in the final anisotropic velocity model. The inversion algorithm was run four times with fixed depths: twice with single events, firstly a central event and secondly a southern event; a group of four events; and a group of eight events. We wanted to understand if the inverted anisotropic parameters are stable for variable groups of events and locations of microseismic events. As shown in the test on the synthetic data set, inversion for depth and anisotropic parameters using a single event is less stable. The two inversions with four and eight events resulted in more mutually consistent values of inverted Thomsen parameters $\epsilon$ and $\delta$ and similar corrections in the origin times of the same events. Inversion of larger numbers of events resulted in very similar (only slightly increased) RMS values, which indicates that our inversion provides realistic values of inverted Thomsen anisotropic parameters. The RMS values of the residuals are very similar to those obtained in the tests on synthetic data sets for the test on the source depth, Thomsen anisotropic parameters and origin time inversion and are between 4-8 RMS ms, which further indicates reliable inversion results. Finally, the values of the inverted Thomsen anisotropic parameters seem to be consistent within the range of the Thomsen parameters obtained from active seismic in shale reservoirs (range between 0.1-0.2 for $\epsilon$ and
Table 4 Inversion results of different groups of microseismic events. The groups of events are separated by thick horizontal lines, i.e., the first group consists in E2_1 only, the third group consist in events E1_1, E2_1, E2_2 and E3_1, etc. The last group contains the inversion results with depth searching. The origin time-shift is the time difference between the isotropic and anisotropic origin times

\begin{tabular}{|c|c|c|c|c|}
\hline Events & $\epsilon$ & $\delta$ & $\begin{array}{l}\text { RMS } \\
\text { (s) }\end{array}$ & $\begin{array}{l}\text { T0 Shift } \\
\text { (s) }\end{array}$ \\
\hline E2_1 & 0.133 & 0.063 & 0.0045 & -0.170 \\
\hline E3_1 & 0.171 & 0.052 & 0.0052 & -0.128 \\
\hline E1_1 & 0.151 & 0.044 & 0.0063 & -0.131 \\
\hline E2_1 & & & 0.0056 & -0.169 \\
\hline E2_2 & & & 0.0062 & -0.170 \\
\hline E3_1 & & & 0.0083 & -0.138 \\
\hline E1_1 & 0.153 & 0.046 & 0.0065 & -0.129 \\
\hline E2_1 & & & 0.006 & -0.168 \\
\hline E2_2 & & & 0.0066 & -0.169 \\
\hline E3_1 & & & 0.0077 & -0.136 \\
\hline E3_2 & & & 0.006 & -0.129 \\
\hline E3_3 & & & 0.0054 & -0.113 \\
\hline E3_4 & & & 0.0048 & -0.111 \\
\hline E4_1 & & & 0.0083 & -0.216 \\
\hline E1_1 & 0.152 & 0.061 & 0.006 & -0.082 \\
\hline E2_1 & & & 0.0046 & -0.104 \\
\hline E2_2 & & & 0.0042 & -0.069 \\
\hline E3_1 & & & 0.0054 & -0.144 \\
\hline E3_2 & & & 0.0059 & -0.088 \\
\hline E3_3 & & & 0.0042 & -0.120 \\
\hline E3_4 & & & 0.0048 & -0.106 \\
\hline E4_1 & & & 0.0082 & -0.206 \\
\hline
\end{tabular}

less than 0.1 for $\delta$ ). Therefore we conclude that inversion of VTI parameters is robust with respect to the numbers of and locations of inverted events in this data set.

In the last group in Table 4, we consider the inversion algorithm that searches the source depths, Thomsen anisotropic parameters and origin times of all events. We constrained the depth search to $300 \mathrm{~m}$ from the depth of the treatment well and reach the final depths of the events not far (less than $200 \mathrm{~m}$ away) from their original depths. The result shows less affected Thomsen anisotropic parameters. All eight events are assigned with initial depths from the initial isotropic velocity model. There is a trade-off between the final inverted depths and the origin time changes (see also Eisner et al. 2010). This is also observed for the synthetics contaminated with noise in Table 3. However, the Thomsen parameters $\epsilon$ and $\delta$ do not seem to be severely affected by this trade-off. Comparing the 
(a)

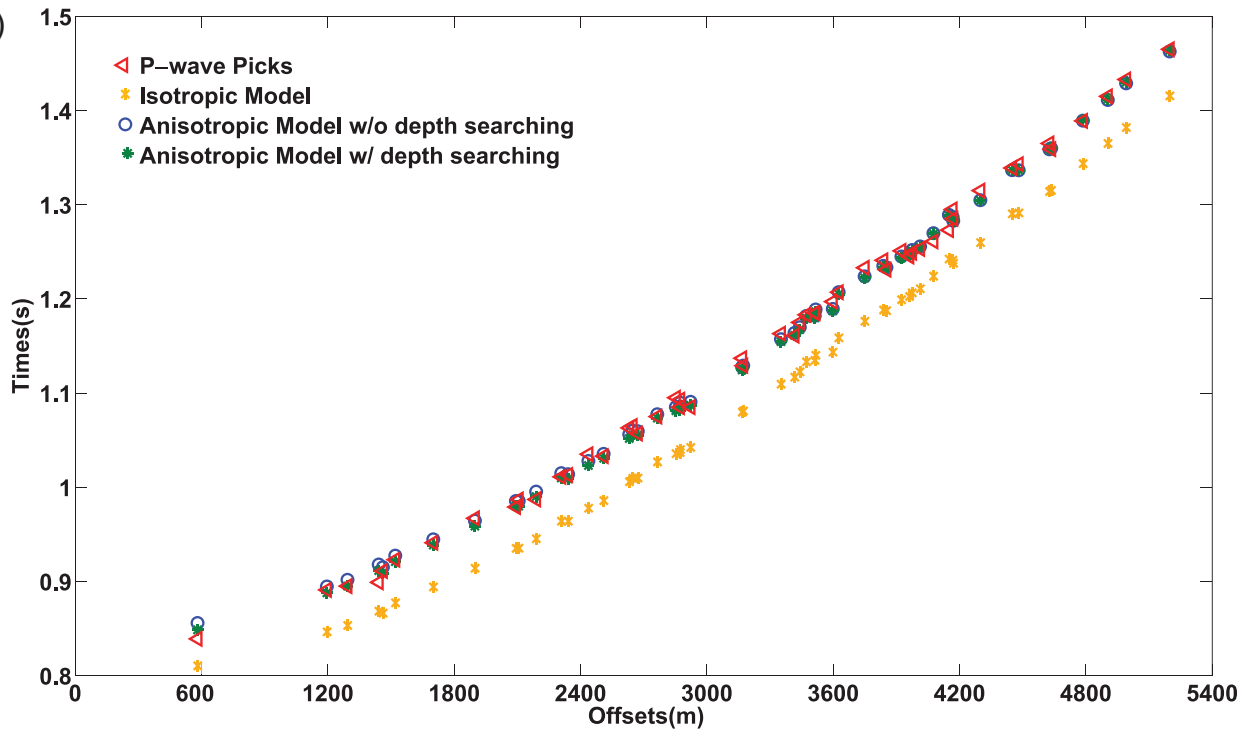

(b)

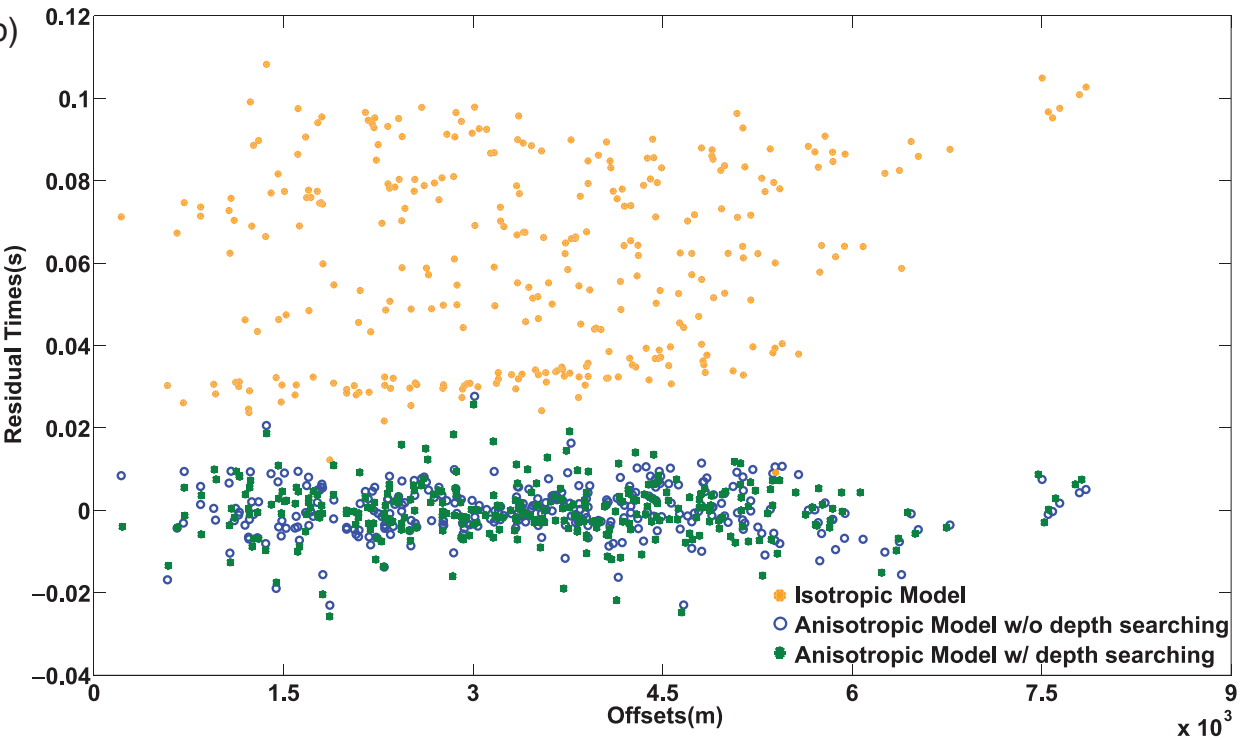

Figure 4 (a) The predicted traveltimes from the isotropic model (yellow) and anisotropic velocity models without (blue) or with (green) depth searching are plotted with the P-wave arrival times (red) from E1_1, an example event from the central part of the array. Note that there is a discrepancy between P-wave picks and the isotropic model that could possibly be eliminated by an origin time-shift. (b) Residuals (picked arrival times minus model traveltimes and origin time) of the isotropic model (yellow) and the anisotropic without (blue) or with (green) depth searching for all eight events.

Thomsen anisotropic parameters from the last two groups of Table 4 one can conclude that the differences in the inverted anisotropic parameters are not significant. Thus, even considering the uncertainty of source depths, we can still achieve a robust estimate of the Thomsen anisotropic parameters.

Figure 4(a) shows an example of arrival times versus offsets for E1_1 through joint inversion of all events from Table 4. The arrival times are compared with traveltimes in an isotropic velocity model and the two anisotropic velocity models in Table $4(\epsilon=0.15$ and $\delta=0.05)$. The isotropic traveltimes are computed at the final depth (obtained from the above inversion). Note that the isotropic traveltimes fit the arrival times much better if the origin time is shifted. Also note that there is a trend of increasing residuals with offsets in the isotropic model. Hence, we obtained a nearly constant shift or origin times between the isotropic and anisotropic velocity models. However, even with the shift the residuals shown in Fig. 4(b) have a much larger scatter for the isotropic velocity model than for the anisotropic velocity models. The isotropic velocity model can also fit the arrival times from approximately the 
same depth if the original isotropic velocity model is scaled up by a factor of 1.22 (this factor was found by a grid search to migrate perforation shots to approximately the right depth). Such scaling of the isotropic velocity model compensates for the anisotropic moveout in small offsets. However, isotropic velocity models cannot fully mimic anisotropic velocity models, especially with receivers at large offsets. The scatter in the time residuals ranges between -0.01 and $0.01 \mathrm{~s}$ for both anisotropic models. This proves that both VTI models can provide a reasonable match with observed arrival times at all offsets without any significant bias or trend.

The above joint inversion for eight microseismic events resulted in a reduced RMS misfit (averaged at $5.6 \mathrm{~ms}$ ) indicating that we can obtain robust estimates of origin times and anisotropic parameters. We obtained Thomsen parameter $\epsilon$ of approximately 0.15 and $\delta$ of 0.05 . This indicates that the reservoir model can be characterized with constant effective Thomsen parameters as the events from the southern part of the array have similar residuals to the events from the central part of the array.

\section{DISCUSSION}

In this study, we approximate the local structure with a $1 \mathrm{D}$ layered medium. It is appropriate given that the majority of the presently explored gas shale sedimentary basins are relatively simple gently dipping structures. We show large-time residuals with offset with isotropic models (Fig. 4b). Although ambiguity between heterogeneity and anisotropy may exist, in our case, the anisotropy effect dominates. If the discrepancy is caused by heterogeneity, the variations of the residuals would have to laterally increase with offset. Instead, in Fig. 4(b), we observe no trend or variation change in the time residuals. Hence the VTI type of anisotropy is the simplest and most reasonable explanation for observed residuals in the isotropic model and heterogeneity does not fit well the residuals. The best-fitting isotropic velocity model explaining the data has a $122 \%$ larger vertical velocity than the measured checkshot model. Such a major discrepancy is not feasible between a well calibrated checkshot model with high-quality vertical velocity and a model for microseismic events. The checkshot model also does not allow for significantly increased or reduced velocities in the isotropic model that could also reduce the trends observed in the residuals.

As explained in the introduction, we invert constant effective anisotropic parameters from the depth of the reservoir to the surface. Although these parameters are physically unrealistic, we are unable to resolve parameters of individ- ual layers as all sources are at approximately the same depth. If only one layer is anisotropic the inversion can be easily adjusted to invert anisotropy only in this layer. Since the microseismic events are noisy, only larger events can be picked individually and used to calibrate the velocity model. Our improved velocity model can in turn be used for stacking and locating the smaller events. As a result, better stacking results can be expected with the calibrated velocity model, which represents the whole area under the array. Therefore, this makes it possible for us to compare the location of small events from one fracture to another for the same region, without introducing the bias of local velocities.

\section{CONCLUSION}

We have developed a method to invert Thomsen anisotropic parameters in a $1 \mathrm{D}$ layered medium from $\mathrm{P}$-wave arrival times of microseismic data (either microseismic events or calibration shots) recorded by a surface array. Our initial velocity model is an isotropic layered model and the Thomsen anisotropic parameters are inverted in a non-linear iterative process. Tests on synthetic data sets show that the method results in correct Thomsen parameters for events with known depths (e.g., calibration shots) and also converges to correct anisotropic parameters for events with unknown depths if the initial estimates of depths are close to correct and residuals are less than $8 \%$ of the total traveltime. The case study results in consistent estimates of anisotropic parameters of $\epsilon$ and $\delta$ and allows locating eight events from about 67 square kilometres $(25$ square miles) in a single velocity model with residuals less than $8 \mathrm{~ms}$. To our best knowledge this is the first study to achieve event locations from multiple hydraulic fracture stimulations (several kilometres apart) with a single velocity model. The effective estimations of Thomsen anisotropic parameters provide an anisotropic velocity model over the region of interest that can be further used for active seismic imaging.

\section{ACKNOWLEDGEMENTS}

We would like to thank Encana and Shell for the release of this data set for publication. We thank also to Vladimir Grechka for his valuable comments.

\section{REFERENCES}

Bakulin A., Liu Y.K., Zdraveva O. and Lyons K. 2010. Anisotropic model building with wells and horizons: Gulf of mexico case study comparing different approaches. The Leading Edge 29, $1450-1460$. 
Bardainne T. and Gaucher E. 2010. Constrained tomography of realistic velocity models in microseismic monitoring using calibration shots. Geophysical Prospecting 58, 739-753.

Bulant P., Eisner L., Psencik I. and Le Calvez J. 2007. Importance of borehole deviation surveys for monitoring of hydraulic fracturing treatments. Geophysical Prospecting 55, 891-899.

Chambers K., Kendall J. and Barkved O. 2010. Investigation of induced microseismicity at valhall using the life of field seismic array. The Leading Edge 29, 290-295.

Duncan P.M. and Eisner L. 2010. Reservoir characterization using surface microseismic monitoring. Geophysics 75, 75A139.

Eisner L., Hulsey B.J., Duncan P., Jurick D., Werner H. and Keller W. 2010. Comparison of surface and borehole locations of induced seismicity. Geophysical Prospecting 58, 809820.

Gei D., Eisner L. and Suhadolc P. 2011. Feasibility of estimation of vertical transverse isotropy from microseismic data recorded by surface monitoring arrays. Submitted to Geophysics.

Grechka V. and Duchkov A.A. 2011. Narrow-angle representations of the phase and group velocities and their applications in anisotropic velocity-model building for microseismic monitoring. Geophysics 76, WC127.

Maxwell S., Bennett L., Jones M. and Walsh J. 2010. Anisotropic velocity modeling for microseismic processing: Part 1 Impact of ve- locity model uncertainty. SEG Technical Program Expanded Abstracts 29, 2130-2134.

Meersman K., Kendall J.-M. and van der Baan M. 2009. The 1998 Valhall microseismic data set: An integrated study of relocated sources,seismic multiplets, and S-wave splitting. Geophysics 74, NO. 5, P. B183-B195.

Prioul R. and Jocker J. 2009. Fracture characterization at multiple scales using borehole images, sonic logs, and walkaround vertical seismic profile. AAPG Bulletin 93, 1503-1516.

Sayers C. 1993. Anelliptic approximations for shales. Journal of Seismic Exploration 2, 319-331.

Sayers C. 1994. The elastic anisotropy of shales. Journal of Geophysical Research 99(B1), 767-774.

Thomsen L. 1986. Weak elastic anisotropy. Geophysics 51 (10), 1954-1966.

Tsvankin I. and Grechka V. 2006. Developments in seismic anisotropy: Treating realistic subsurface models in imaging and fracture detection. CSEG Recorder 31 (special edition), 43-46.

Vernik L. 2008. Anisotropic correction of sonic logs in wells with large relative dip. Geophysics 73, E1.

Wuestefeld A., Al Harrasi O., Verdon J.P., Wookey J. and Kendall J.M. 2010. A strategy for automated analysis of passive microseismic data to image seismic anisotropy and fracture characteristics. Geophysical Prospecting 58, 755-773. 